\title{
Adsorption of hydrogen peroxide on functionalized mesoporous silica surfaces
}

\author{
Dawid Lewandowski • Dawid Bajerlein • \\ Grzegorz Schroeder
}

Received: 7 November 2013/Accepted: 19 March 2014/Published online: 8 April 2014

(c) The Author(s) 2014. This article is published with open access at Springerlink.com

\begin{abstract}
MCM-41 and MSU-H mesoporous silicas were successfully functionalized with hydrogen bonds forming organic moieties, which have been proven by elemental analysis. Both moieties, based on oxygen and nitrogen containing groups, were introduced with high efficiencythe amount of carbon in all cases exceeded $10 \%$ and the elemental ratios suggest binding to the surface through two or three $\mathrm{Si}-\mathrm{O}-\mathrm{Si}$ bonds. Hydrogen peroxide adsorption was conducted in its aqueous solutions and the amount adsorbed was determined using the ferric thiocyanate method. Results are presented as a function of hydrogen peroxide concentration in aqueous solution from 5 to $30 \%$. Both functionalized silicas show increased adsorption capacity when compared with that of their unfunctionalized analogues. The surface modified with nitrogen-based organic moiety revealed better adsorption properties as well as higher resistance against oxidation. MSU-H silica, due to its larger pore diameter, provides more space to bind hydrogen peroxide molecules and thus was found to have higher adsorption capacity: it adsorbed up to four times more hydrogen peroxide than MCM-41.
\end{abstract}

Keywords MCM-41 MSU-H $\cdot$ Hydrogen peroxide . Adsorption

\section{Introduction}

Hydrogen peroxide is a very useful oxidizer that has been employed in many different fields, such as chemistry of

D. Lewandowski $(\bowtie) \cdot$ D. Bajerlein · G. Schroeder

Department of Chemistry, Adam Mickiewicz University,

Umultowska 89b, 61-614 Poznan, Poland

e-mail: dawid_le@amu.edu.pl atmosphere [1], autodissociation dynamics [2, 3], and bleaching [4]. It is reasonably stable, readily available, inexpensive, and generates only water as a by-product [5]. Although its molecule is similar to water, it has not been much studied in a pure state because of its instability. However, like water, it forms hydrogen bonds with nitrogen- and oxygen-containing compounds [6, 7].

To avoid the stability issues, hydrogen peroxide can be transformed into a number of complexes with organic compounds. Urea-hydrogen peroxide complex (UHP) has already been used in many oxidation processes [8-11] as a source of anhydrous hydrogen peroxide. It is a stable and inexpensive solid that has been first prepared by Tanatar [12] and now is sold by a number of chemical manufacturers. Its crystals are composed of molecules of urea and hydrogen peroxide in 1:1 ratio, connected through multiple hydrogen bonds [13]. Hydrogen peroxide forms also complexes with melamine [14] and DABCO-di- $N$-oxide [15]. Some polymer-supported systems containing hydrogen peroxide are known as well. The most prominent among them is polyvinylpyrrolidone-hydrogen peroxide complex $\left(\mathrm{PVP}-\mathrm{H}_{2} \mathrm{O}_{2}\right)$, introduced by Pourali and Ghanei [16] that has already been successfully used for iodination of aromatic compounds as well as for oxidation of $\alpha, \beta$-enones [17]. It can be easily prepared from $30 \%$ aqueous solution of hydrogen peroxide and polyvinylpyrrolidone $\mathrm{K}-30$, it contains up to $23 \%$ of hydrogen peroxide by weight [18] and is insoluble in organic solvents [19]. It is stable for several months while stored in a refrigerator, without losing its weight or activity [16]. Hydrogen peroxide can also be encapsulated in different silica xerogel systems to serve as an oxidant in the presence of metal catalyst [20-22] or without it, with a hydrogen peroxide content up to $68 \%$ [23].

Mesoporous materials developed in the 1990s, show numerous advantages: large surface area $\left(\sim 1,000 \mathrm{~m}^{2} / \mathrm{g}\right)$, 
uniform and controllable pore size, large pore volume $\left(\sim 1 \mathrm{~cm}^{3} / \mathrm{g}\right)$, narrow particle size, open pore structures [24], chemical and biological stability, and possibility of control of surface functionalization, which makes them very useful in a wide variety of applications such as adsorption, catalysis, ion exchange, sensing [25], chromatography [26], delivery carriers, and controlled drug/gene release [27]. There are three general paths to achieve surface functionalization of mesoporous silica surface [28]: postsynthetic functionalization (the so-called "grafting") and two methods involved in the silica preparation process-co-condensation and condensation of bridged organosilica precursors forming periodic mesoporous organosilicas. The use of grafting method usually preserves the mesostructure of the starting silica phase, but the distribution of organic moieties anchored to the silica surface is solvent dependent [29] and sometimes leads to pores blockage [30]. Another advantage of this method is that it can be applied to a diversity of organic molecules, from small ones like aminopropyltrimethoxysilane [29] or compounds used in our experiment, to large moieties as dendrimers [31] and fullerenes [32]. Co-condensation is a convenient direct method of synthesis when the total amount of the organic part is supposed to be rather small. It provides better homogeneity of organic moieties, but can alter the matrix structure leading sometimes to totally disordered products [28]. It also allows the use of extraction as the only method for surfactant removal, as calcination could destroy organic compounds. After pioneering works of the groups of Mann and co-workers [33] and MacQuarrie [34], many different organically modified mesoporous silicas have been prepared by co-condensation, including those modified with alkyl, thiol, vinyl/allyl [35], amino, cyano/isocyano [36], alkoxy [37], organophosphine, and aromatic groups [38]. Periodic mesoporous organosilicas (PMOs) are materials with modified matrices but preserved organized pore system and narrow pore size distribution. They were first synthesized in 1999 by three groups working independently [39-41]. Until now, various PMOs are known: ethane- [39], ethene- [40], benzene[42], bisphenyl-bridged [43], etc. They have found their application in chromatography [44] and catalysis [45].

The study was conducted to verify the ability of organic moieties grafted onto the mesoporous silicas of MCM-41 and MSU-H types to adsorb hydrogen peroxide from its aqueous solutions at different concentrations. Silica was chosen for the experiment, as it can be easily functionalized and has native silanol groups, able to form desired hydrogen bonds. Furthermore, both types of silica used differ in the pore diameter and noticeably in the surface area. We have used the method of postsynthetic modification to anchor nitrogen- and oxygen-containing groups that are able to form hydrogen bonds as grafting method offers higher coverage efficiency than direct synthesis methods and do not influence the order of mesoporous silica structure. Nitrogen-based compound (1-[3-(triethoxysilyl)propyl]urea) with urea-like moiety was chosen to permit comparison of the ability of hydrogen peroxide binding of this moiety and pure urea. Oxygen-based compound ( $N$-(3-triethoxysilylpropyl)gluconamide) has many hydroxyl groups, and it was chosen to verify their influence on binding hydrogen peroxide.

This study is the first on hydrogen peroxide adsorption (not decomposition) on the surface of organically modified mesoporous silica. It is supposed to be the basis for future articles covering the presented issue.

\section{Experimental details}

\section{Materials}

Mesoporous silicas-MCM-41 (2.1-2.7 nm in diameter and $1,000 \mathrm{~m}^{2} / \mathrm{g}$ surface area) and MSU-H $(\sim 7.1 \mathrm{~nm}$ in diameter and $750 \mathrm{~m}^{2} / \mathrm{g}$ surface area), $30 \%$ stock solution of hydrogen peroxide and 3-(triethoxysilyl)propyl isocyanate used for the synthesis of 1-[3-(triethoxysilyl)propyl]urea (1) were all purchased from Sigma-Aldrich, and $N$-(3-triethoxysilylpropyl)gluconamide (2) was purchased from ABCR GmbH \& Co. KG. The structures of compounds used are presented in Fig. 1. Water used for the preparation of diluted hydrogen peroxide solutions had been degassed and deionized.

\section{Preparation of reagents}

Both mesoporous silicas had been prepared for grafting by activation in boiling mixture of nitric acid and water (1:1) for a few hours, filtered off, washed by large amount of water, and dried in $60{ }^{\circ} \mathrm{C}$ for several hours.

A portion of $2 \mathrm{~g}$ of 3-(triethoxysilyl)propyl isocyanate was dissolved in $20 \mathrm{ml}$ of acetonitrile and reacted with dry gaseous ammonia. The substrates reacted vigorously, so the solution was mixed and the flask was held in a cool water bath. After $1 \mathrm{~h}$, the reaction was stopped, and both ammonia and acetonitrile were evacuated under vacuum. Gelatinous, quickly crystallizing solid, identified as $N$-[3(triethoxysilyl)propyl]urea, was obtained. Figures 2, 3, and 4 present the spectra obtained for the isolated compound.

${ }^{1} \mathrm{H}$ NMR spectrum was taken on an NMR Varian VNMR-S $400 \mathrm{MHz}$ spectrometer at $298 \mathrm{~K}$ with TMS as a reference. ${ }^{1} \mathrm{H} \quad \mathrm{NMR}\left(400 \mathrm{MHz}, \mathrm{CDCl}_{3}\right), \delta: 0.62-0.67$ (t, 2H), 1.20-1.25 (t, 9H), 1.57-1.66 (quin, 2H), 3.11-3.17 (q, 2H), 3.79-3.85 (q, 6H), 4.74-4.77 (s, 2H), 5.20-5.28 (s, 1H). 
<smiles>CCO[Si](CCCNC(N)=O)(COC)OCC</smiles>

1<smiles>CCO[Si](CCCNC(=O)[C@H](O)[C@H](O)[C@H](O)[C@H](O)CO)(COC)OCC</smiles>

2

Fig. 1 Molecules grafted on selected mesoporous silicas surface-1 stands for 1-[3-(triethoxysilyl)propyl]urea and 2 is $N$-(3triethoxysilylpropyl)gluconamide

Fig. $2{ }^{1} \mathrm{H}$ NMR spectrum of $\mathrm{N}$-[3-(triethoxysilyl)propyl]urea

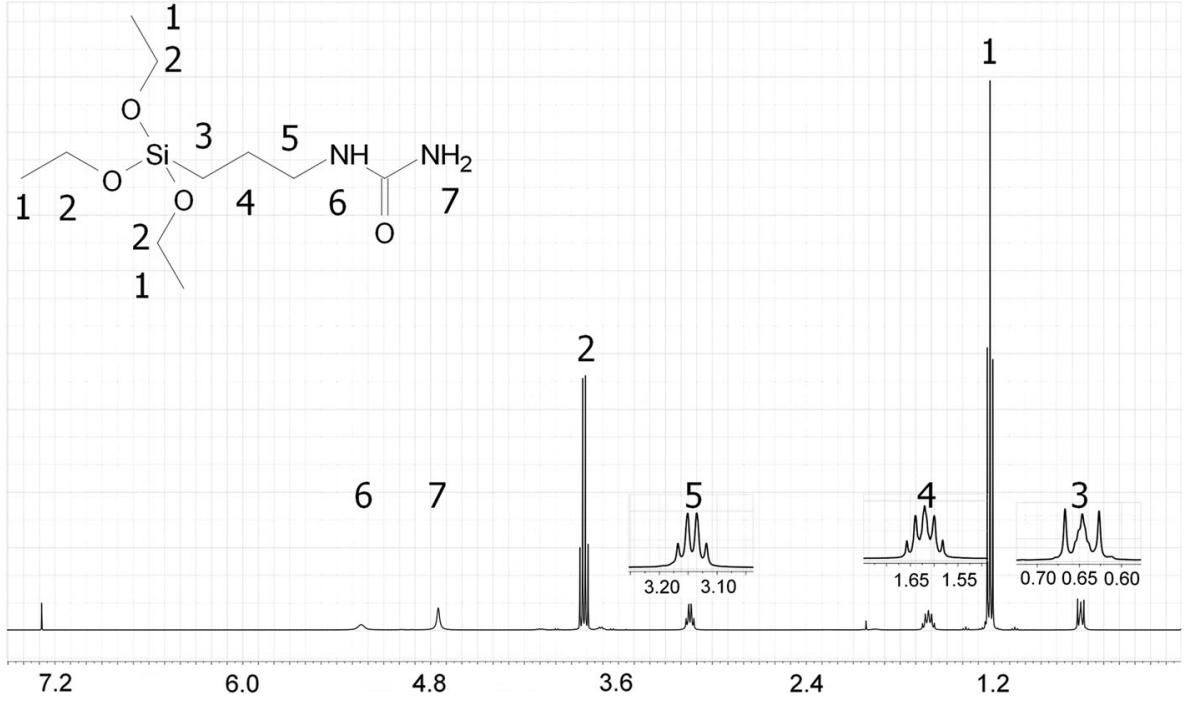

Fig. 3 ESI MS spectrum of $N$-[3-(triethoxysilyl)propyl]urea

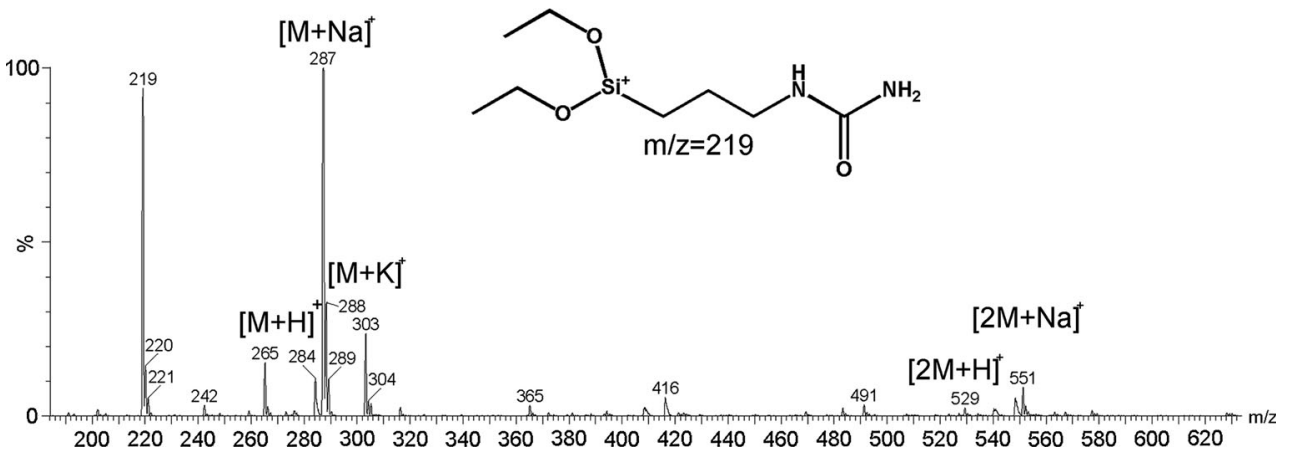

ESI MS spectrum was obtained on a Waters/Micromass ZQ Mass Detector using $10^{-3} \mathrm{M}$ methanol solution. ESI analysis parameters were the following: cone voltage$30 \mathrm{~V}$, capillary voltage $-3 \mathrm{kV}$, ion source temperature$393 \mathrm{~K}$, desolvation temperature- $573 \mathrm{~K}$, and nitrogen was used as the nebulizing and desolving gas at flow rate of 80 1/min. ESI MS m/z: $[\mathrm{M}+\mathrm{H}]^{+} 265,[\mathrm{M}+\mathrm{Na}]^{+} 287$, $[\mathrm{M}+\mathrm{K}]^{+} 303$.

IR spectrum was obtained on a Bruker FT-IR IFS 66/s spectrometer using $1.2 \mathrm{mg}$ of the compound mixed with $200 \mathrm{mg}$ of $\mathrm{KBr}$ and formed into a pellet. IR: $3670-3440 \mathrm{~cm}^{-1} \mathrm{~N}-\mathrm{H}$ amide stretching, $2975-2885 \mathrm{~cm}^{-1}$
$\mathrm{C}-\mathrm{H}$ alkyl stretching, $1668 \mathrm{~cm}^{-1} \mathrm{C}=\mathrm{O}$ amide stretching, $1593,1526 \mathrm{~cm}^{-1} \mathrm{~N}-\mathrm{H}$ amide bending.

Preparation of modified silica

The procedure of modifying both silicas with each of grafting reagents used was as follows: $250 \mathrm{mg}$ of the grafting reagent was dissolved in $10 \mathrm{ml}$ of toluene, and then $250 \mathrm{mg}$ of unmodified mesoporous silica was suspended. The mixture was stirred under reflux for $6 \mathrm{~h}$, cooled to room temperature, filtered off, washed with pure toluene, and dried in $60{ }^{\circ} \mathrm{C}$ for several hours. 


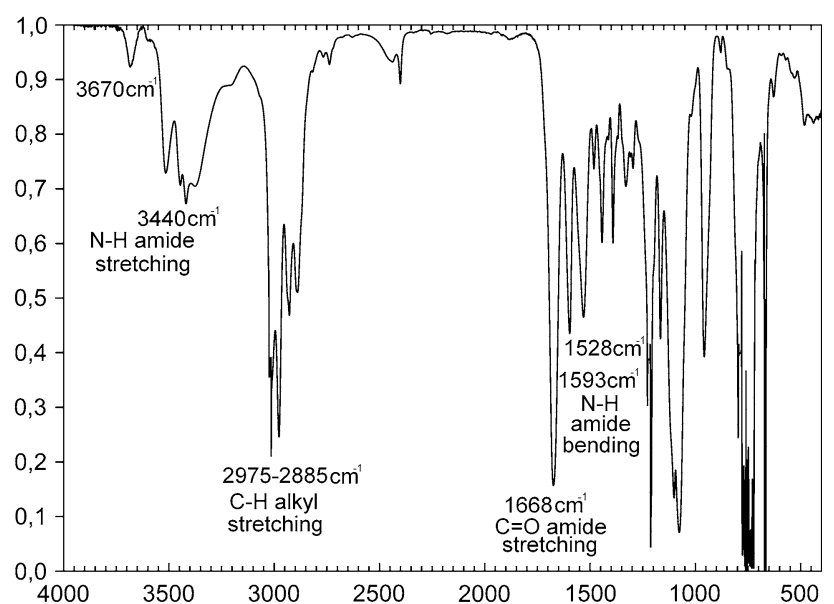

Fig. 4 IR spectrum of $N$-[3-(triethoxysilyl)propyl]urea

Hydrogen peroxide adsorption analysis

A series of hydrogen peroxide solutions with concentrations increasing from 5 to $30 \%$ and pure, deionized, and degassed water were prepared for both unmodified and modified silica systems. Each system was composed of $35 \mathrm{mg}$ of a selected silica suspended in $6 \mathrm{ml}$ of hydrogen peroxide solution in a phial and stirred with a magnetic stirrer at room temperature for $24 \mathrm{~h}$. Then, the phial contents were filtered off, washed with decent amount of water, and dried in $50{ }^{\circ} \mathrm{C}$ for $2 \mathrm{~h}$ to avoid hydrogen peroxide decomposition. The amount of hydrogen peroxide adsorbed was determined using the ferric thiocyanate method [46]. Before absorbance measurement on an UV-Vis Agilent 8453 Spectrophotometer, the silica suspensions were centrifuged to avoid
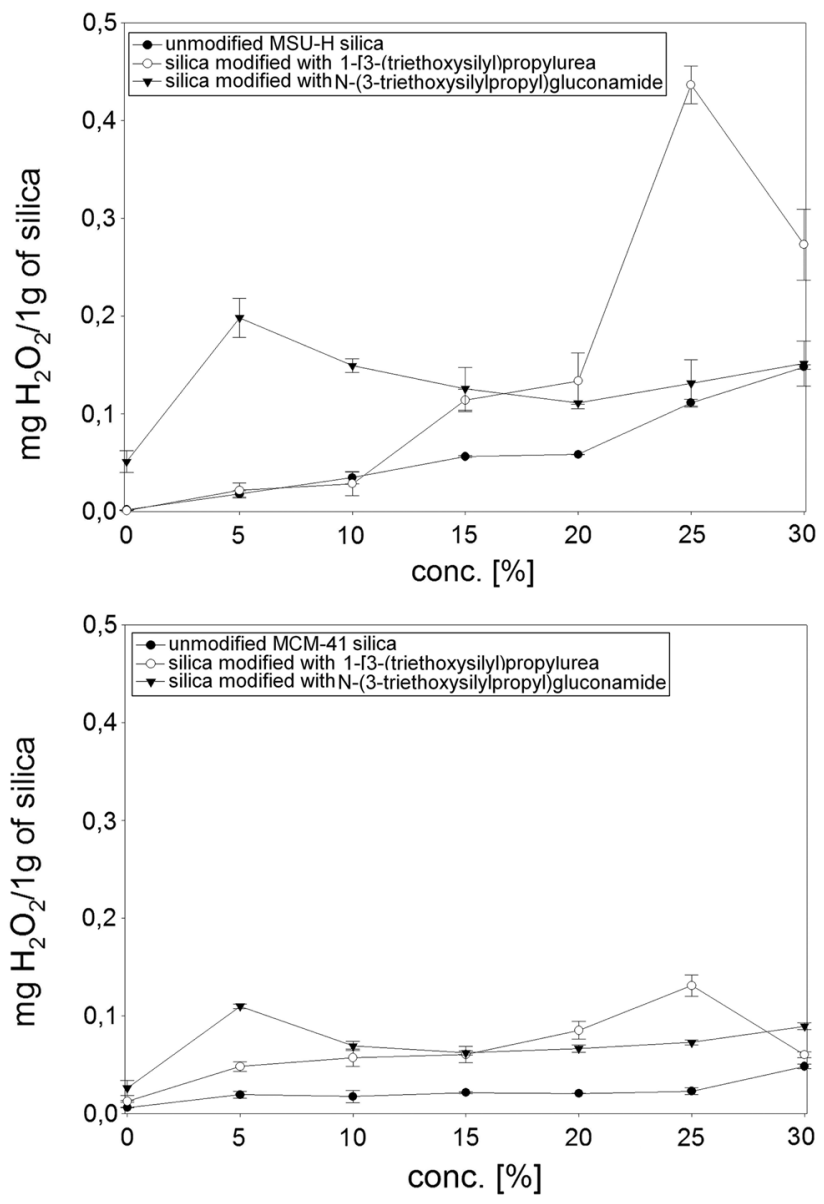

Fig. 5 Amount of hydrogen peroxide adsorbed on each functionalized silica as a function of hydrogen peroxide concentration in aqueous solution

disturbances caused by silica particles, and only clear solution from over the precipitate was taken for measurement.
Table 1 Comparison of grafting efficiency for all obtained systems

\begin{tabular}{llllll}
\hline Grafting compound & $\begin{array}{l}\text { Mesoporous } \\
\text { silica type }\end{array}$ & \multicolumn{2}{l}{ Mass percentages of selected elements } & \multirow{2}{*}{$\begin{array}{l}\text { Mass ratios } \\
\text { C:H:N }\end{array}$} \\
\cline { 3 - 4 } & & $\begin{array}{l}\text { Carbon } \\
(\%)\end{array}$ & $\begin{array}{l}\text { Hydrogen } \\
(\%)\end{array}$ & $\begin{array}{l}\text { Nitrogen } \\
(\%)\end{array}$ & \\
\hline $\begin{array}{l}\text { 1-[3-(Triethoxysilyl) } \\
\text { propyl]urea }\end{array}$ & MCM-41 & 11.70 & 3.044 & 4.371 & $2.68: 0.70: 1$ \\
& & 11.74 & 3.065 & 4.276 & $2.75: 0.72: 1$ \\
& MSU-H & 12.31 & 3.160 & 6.310 & $1.95: 0.50: 1$ \\
& & 12.27 & 3.190 & 6.250 & $1.96: 0.51: 1$ \\
$N$-(3-Triethoxysilylpropyl) & MCM-41 & 15.68 & 3.786 & 1.925 & $8.14: 1.97: 1$ \\
gluconamide & & 15.71 & 3.954 & 1.969 & $7.98: 2.01: 1$ \\
& MSU-H & 16.17 & 3.498 & 1.939 & $8.34: 1.80: 1$ \\
& & 16.21 & 3.534 & 1.877 & $8.64: 1.88: 1$ \\
Pure MCM-41 & & 0.030 & 0.832 & 0.034 & $0,88: 24.54: 1$ \\
& & 0.043 & 0.879 & 0.043 & $1.00: 20.44: 1$ \\
Pure MSU-H & & 0.089 & 1.215 & 0.044 & $2.02: 27.61: 1$ \\
& & 0.093 & 1.242 & 0.012 & $7.75: 103.50: 1$ \\
\hline
\end{tabular}




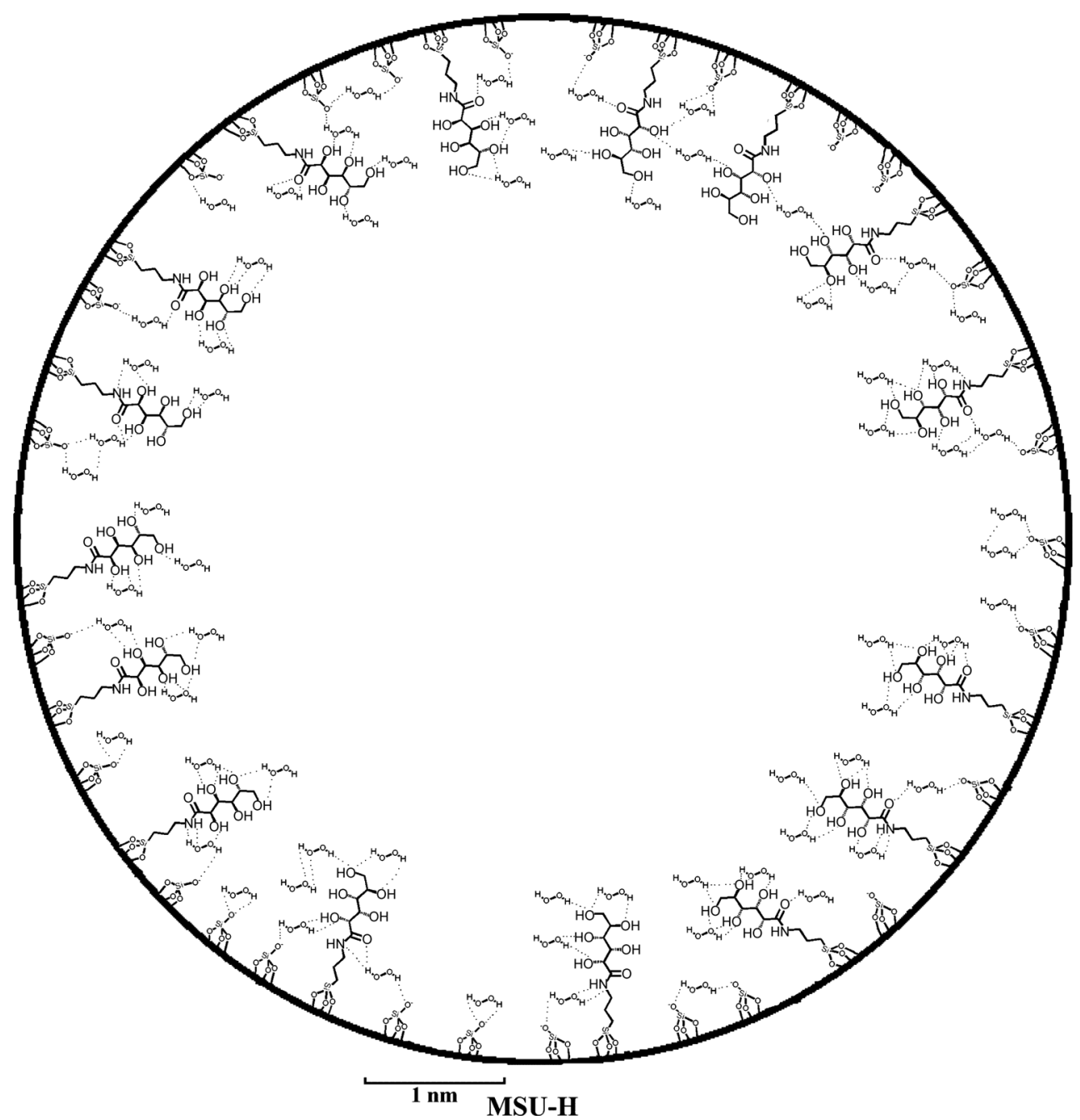

Fig. 6 Cross-section of MSU-H silica, functionalized with $\mathrm{N}$-(3-triethoxysilylpropyl)gluconamide, explaining the possible organization of grafted molecules and hydrogen peroxide adsorbed in the pores. Channel diameter and size of the molecules match

\section{Results and discussion}

Elemental analysis

Elemental analysis for carbon, hydrogen, and nitrogen content allowed determination of the amounts of grafting reagents that had reacted with the silica surface. Results are presented in Table 1.

The presence of carbon in pure silicas can be a residue of surfactant used in the manufacturing process. Nitrogen observed in pure silica can be either adsorbed from the air or a surfactant residue, and hydrogen is a component of silanol groups densely covering the surface. The number of $\mathrm{Si}-\mathrm{O}-\mathrm{Si}$ bonds linking the grafted molecules to the surface could be easily calculated from the carbon:nitrogen ratio as hydrogen balance was more difficult to calculate. Hydrogen was removed from the surface silanol groups and added with grafted molecules in the amount depending on the total amount of the given compound grafted, hydrolysis of its alkoxy-groups, and the amount of $\mathrm{Si}-\mathrm{O}-\mathrm{Si}$ links formed.

The unbound 1-[3-(triethoxysilyl)propyl]urea of the molecular formula $\mathrm{C}_{10} \mathrm{H}_{24} \mathrm{~N}_{2} \mathrm{O}_{4} \mathrm{Si}$ and the carbon:nitrogen ratio equal to 4.29:1 can be linked through one (with one ethoxy-group removed), two, or three $\mathrm{Si}-\mathrm{O}-\mathrm{Si}$ links with carbon:nitrogen ratios equal to $3.43: 1,2.57: 1$, and 1.71:1, respectively. The carbon:nitrogen ratios obtained for this compound suggest binding through two $\mathrm{Si}-\mathrm{O}-\mathrm{Si}$ links, when grafted on MCM-41, and through two or three (two links:three links ratio is 0.29:0.71) links, when grafted on MSU-H silica. Similar analysis conducted for binding of $\mathrm{N}$-(3-triethoxysilylpropyl)gluconamide led to the following results: unbound compound of the molecular formula $\mathrm{C}_{15} \mathrm{H}_{33} \mathrm{NO}_{9} \mathrm{Si}$ and the carbon:nitrogen ratio equal to 
12.86:1 can be linked through one, two, or all three links with carbon:nitrogen ratios equal to $11.14: 1,9.43: 1$, and 7.71:1, respectively. The carbon:nitrogen ratios for this compound suggest binding to the surface of both silicas through two or three $\mathrm{Si}-\mathrm{O}-\mathrm{Si}$ links (for MCM-41 two links:three links ratio is 0.21:0.79 and for MSU-H 0.45:0.55)

The silicas obtained contain large amounts of grafted compounds on their surface, comparable to those of simple organic compounds grafted in the previous studies on functionalization of mesoporous surfaces [47, 48], that could be estimated from the amount of nitrogen as its content is not dependent on the binding degree. 1-[3-(Triethoxysilyl)propyl]urea accounts for $32.11 \%$ of total mass of modified MCM-41 and $41.71 \%$ of MSU-H, which is 1.37 molecules $/ \mathrm{nm}^{2}$ of MCM-41 surface and 3.09 molecules $/ \mathrm{nm}^{2}$ of MSU-H surface. $N$-(3-Triethoxysilylpropyl)gluconamide accounts for $44.51 \%$ of total mass of modified MCM-41 and $45.32 \%$ of MSU-H, which is 1.51 molecules $/ \mathrm{nm}^{2}$ of MCM-41 surface and 2.00 molecules $/ \mathrm{nm}^{2}$ of MSU-H. These results show clearly that the pore size is an important factor, influencing the total amount of compounds grafted.

\section{Hydrogen peroxide adsorption analysis}

The graphs presented in Fig. 5 for different mesoporous silicas show clearly that surface modification influenced the amounts of hydrogen peroxide adsorbed on its surface, which after modification increased several times. The adsorption capacity of silicas modified with 1-[3-(triethoxysilyl)propyl]urea was increasing nonlinearly up to a hydrogen peroxide concentration of $25 \%$, and then it dropped to values similar to those obtained for unmodified silica, probably because of the destruction of organic moiety anchored to the silica surface (loss of the "urea" part makes it unable to form hydrogen bonds). The silicas modified with $N$-(3-triethoxysilylpropyl)gluconamide have their maximum adsorption at $5 \%$ concentration of hydrogen peroxide, and then it decreases slightly to become almost equal to the adsorption of unmodified silica at $30 \%$ hydrogen peroxide concentration. A large number of hydroxyl groups included in gluconamide moiety are oxidized, even at lower concentrations, and partially lose their ability to form hydrogen bonds (carbonyl group, without a hydrogen atom, can only be an electron donor). The average contents of carbon, hydrogen, and nitrogen for samples bathed in a $30 \%$ solution of hydrogen peroxide are, respectively, $7.468,2.261$, and $3.897 \%$. The carbon:nitrogen ratio is much smaller than that for the unoxidized sample, which proves a major destruction to the organic part and the loss of ability to bind hydrogen peroxide. Small increase in the amount of hydrogen peroxide

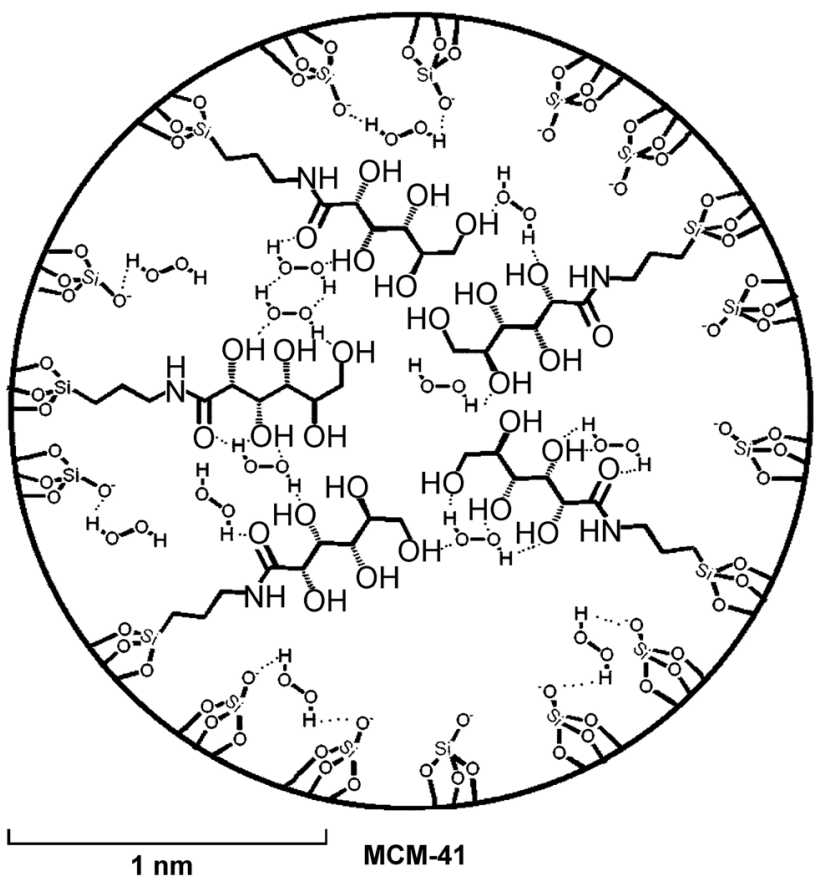

Fig. 7 Cross-section of MCM-41 silica, functionalized with $\mathrm{N}$-(3triethoxysilylpropyl)gluconamide, explaining the possible organization of grafted molecules and hydrogen peroxide adsorbed in the pores. Channel diameter and size of the molecules match

adsorbed at 25 and $30 \%$ concentrations in comparison to that adsorbed from hydrogen peroxide solutions of concentrations 20 and $25 \%$ may be caused by adsorption on the uncovered part of the silica surface.

Also, MSU-H silica was found to adsorb much higher amounts of hydrogen peroxide, although it has smaller surface area. The influence of pore diameter on the amounts of hydrogen peroxide adsorbed is also indicated by the results obtained for each silica separately. The maximum amounts of hydrogen peroxide for MCM-41 are similar because of available space limitations. Grafted molecules occupy most of the pore volume, so there is not much space to bind $\mathrm{H}_{2} \mathrm{O}_{2}$ and its diffusion may be impaired. MSU-H provides much more space for both grafted organic moieties and hydrogen peroxide adsorbed as can be seen comparing Figs. 6 and 7, and the amount of $\mathrm{H}_{2} \mathrm{O}_{2}$ is only limited by the binding abilities of grafted molecules.

The amount of hydrogen peroxide adsorbed on unmodified silica surface almost linearly increases with increasing hydrogen peroxide concentration. This phenomenon can be explained by formation of hydrogen bonds between free silanol groups and hydrogen peroxide. In the experimental conditions, almost all of silanol groups were ionized as mesoporous silanol groups existed in two forms, $\mathrm{Si}-(\mathrm{OH})_{2}$ and $\mathrm{Si}-(\mathrm{OH})$ with $\mathrm{p} K_{\mathrm{a}}$ values, respectively, 8.2 and 2.0 [49]. 


\section{Conclusions}

Two different molecules able to form hydrogen bonds were successfully grafted on the surface of two different mesoporous silicas of MCM-41 and MSU-H types. The amounts of hydrogen peroxide adsorbed on their surface were measured, and MSU-H sample was found to have a higher adsorption capacity, as about 2-3 times more hydrogen peroxide got adsorbed on its surface. Unmodified silica showed also some adsorption abilities dependent on the concentration of hydrogen peroxide solution. Functionalized surface was not resistant enough and the compounds deposited underwent oxidation, changing the silicas adsorption properties in higher hydrogen peroxide concentrations. Increased amount of hydroxyl groups introduced with $\mathrm{N}$-(3-triethoxysilylpropyl)gluconamide indeed has enhanced the adsorption of hydrogen peroxide. Although the content of hydrogen peroxide bound by the systems obtained was small in comparison with that of PVP or urea complexes, results of this study can initiate further development in this field.

Acknowledgments The authors would like to thank the National Science Center of Poland (Grant No. 2011/03/B/ST5/01573) for financial support.

Open Access This article is distributed under the terms of the Creative Commons Attribution License which permits any use, distribution, and reproduction in any medium, provided the original author(s) and the source are credited.

\section{References}

1. Wayne RP (1991) Chemistry of atmospheres. Clarendon Press, Oxford

2. Gericke K-H, Klee S, Comes FJ, Dixon RN (1986) Dynamics of $\mathrm{H}_{2} \mathrm{O}_{2}$ photodissociation: $\mathrm{OH}$ product state and momentum distribution characterized by sub-Doppler and polarization spectroscopy. J Chem Phys 85:4463-4479

3. Docker MP, Hodgson A, Simons JP (1986) Photodissociation of $\mathrm{H}_{2} \mathrm{O}_{2}$ at $248 \mathrm{~nm}$ : translational anisotropy and oh product state distributions. Chem Phys Lett 128:264-269

4. Hart PW, Rudie AW (2012) The bleaching of pulp, 5th edn. Tappi Press, Norcross

5. Kureshy RI, Khan NH, Abdi SHR, Patel ST, Jasra RV (2001) Enantioselective epoxidation of non-functionalised alkenes using a urea-hydrogen peroxide oxidant and a dimeric homochiral Mn(III)-Schiff base complex catalyst. Tetrahedron Asymmetry 12:433-437

6. Goebel JR, Ault BS, Del Bene JE (2001) Matrix isolation and ab initio study of $1: 1$ hydrogen-bonded complexes of $\mathrm{H}_{2} \mathrm{O}_{2}$ with $\mathrm{NH}_{3}$ and $\mathrm{N}\left(\mathrm{CH}_{3}\right)_{3}$. J Phys Chem A 105:6430-6435

7. Goebel J, Ault BS, Del Bene JE (2000) Matrix isolation and ab initio study of the hydrogen-bonded complex between $\mathrm{H}_{2} \mathrm{O}_{2}$ and $\left(\mathrm{CH}_{3}\right)_{2} \mathrm{O}$. J Phys Chem A 104:2033-2037

8. Boehlow TR, Spilling CD (1996) The regio- and stereo-selective epoxidation of alkenes with methyl trioxorhenium and ureahydrogen peroxide adduct. Tetrahedron Lett 37:2717-2720
9. Hasaninejad A, Zolfigol MA, Chehardoli G, Mokhlesi M (2010) Molybdatophosphoric acid as an efficient catalyst for the catalytic and chemoselective oxidation of sulfides to sulfoxides using urea hydrogen peroxide as a commercially available oxidant. J Serb Chem Soc 75:307-316

10. Varma RS, Naicker KP (1999) The urea-hydrogen peroxide complex: solid-state oxidative protocols for hydroxylated aldehydes and ketones (Dakin reaction), nitriles, sulfides, and nitrogen heterocycles. Org Lett 1:189-192

11. Caron S, Do NM, Sieser JE (2000) A practical, efficient, and rapid method for the oxidation of electron deficient pyridines using trifluoroacetic anhydride and hydrogen peroxide-urea complex. Tetrahedron Lett 41:2299-2302

12. Tanatar S (1906) J Russ Phys Chem Soc 40L:376

13. Lu C-S, Hughes EW, Giguère PA (1941) The crystal structure of the urea-hydrogen peroxide addition compound $\mathrm{CO}\left(\mathrm{NH}_{2}\right)_{2} \mathrm{H}_{2} \mathrm{O}_{2}$. J Am Chem Soc 63:1507-1513

14. Chehardoli G, Zolfigol MA (2009) Melamine hydrogen peroxide (MHP): novel and efficient reagent for the chemo- and homoselective and transition metal-free oxidation of thiols and sulfides. Phosphorus Sulfur Silicon Relat Elem 185:193-203

15. Zolfigol MA, Salehi P, Mallakpour SE, Torabi M (2003) 1,4Diazabicyclo[2.2.2] octane 1,4-bis(oxide)-bis(hydrogen peroxide) $/ \mathrm{MCl}_{\mathrm{x}}$ as a novel heterogeneous system for the oxidation of urazoles under mild conditions. Bull Chem Soc Jpn 76:1673-1674

16. Pourali AR, Ghanei M (2006) Direct Iodination of aromatic compounds with polyvinylpyrrolidone supported hydrogen peroxide $\left(\mathrm{PVP}-\mathrm{H}_{2} \mathrm{O}_{2}\right)$ and potassium iodide or molecular iodine. Chin J Chem 24:1077-1079

17. Pourali AR, Ghanei M (2006) Efficient epoxidation of $\alpha$, $\beta$ enones with polyvinylpyrrolidone supported hydrogen peroxide (PVP- $\mathrm{H}_{2} \mathrm{O}_{2}$ ). Bull Korean Chem Soc 27:1674-1676

18. Merianos JJ (1991) Anhydrous complexes of PVP and hydrogen peroxide. US Patent 5,008,093

19. Ghorbani-Choghamarani A, Azadi G (2011) Polyvinylpolypyrrolidone-supported hydrogen peroxide $\left(\mathrm{PVP}-\mathrm{H}_{2} \mathrm{O}_{2}\right)$, silica sulfuric acid and catalytic amounts of ammonium bromide as green, mild and metal-free oxidizing media for the efficient oxidation of alcohols and sulfides. JICS 8:1082-1090

20. Kochkar H, Figueras F (1997) Synthesis of hydrophobic $\mathrm{TiO}_{2}-$ $\mathrm{SiO}_{2}$ mixed oxides for the epoxidation of cyclohexene. J Catal 171:420-430

21. Barbosa GN, MacLeod TCO, Guedes DFC, Assis MD, Oliveira HP (2008) Preparation, characterization and catalytic studies of $\mathrm{V}_{2} \mathrm{O}_{5}-\mathrm{SiO}_{2}$ xerogel composite. J Sol-Gel Sci Technol 46:99-105

22. Neumann R, Levin-Elad M (1997) Metal oxide $\left(\mathrm{TiO}_{2}, \mathrm{MoO}_{3}\right.$, $\mathrm{WO}_{3}$ ) substituted silicate xerogels as catalysts for the oxidation of hydrocarbons with hydrogen peroxide. J Catal 166:206-217

23. Bednarz S, Ryś B, Bogdał D (2012) Application of hydrogen peroxide encapsulated in silica xerogels to oxidation reactions. Molecules 17:8068-8078

24. Popat A, Liu J, Hu Q, Kennedy M, Peters B, Lu GQM, Qiao SZ (2012) Adsorption and release of biocides with mesoporous silica nanoparticles. Nanoscale 4:970-975

25. Vallet-Regi M, Ramila A, Real RPd, Perez-Pariente J (2001) A new property of MCM-41: drug delivery system. Chem Mater 13:308-311

26. Mal NK, Fujiwara M, Tanaka Y (2003) Photocontrolled reversible release of guest molecules from coumarin-modified mesoporous silica. Nature 421:350-353

27. He Q, Gao Y, Zhang L, Zhang Z, Gao F, Ji X, Li Y, Shi J (2011) A pH-responsive mesoporous silica nanoparticles-based multidrug delivery system for overcoming multi-drug resistance. Biomaterials 32:7711-7720 
28. Hoffmann F, Cornelius M, Morell J, Froba M (2006) Silica-based mesoporous organic-inorganic hybrid materials. Angew Chem Int Ed 45:3216-3251

29. Salmio H, Bruhwiler D (2007) Distribution of amino groups on a mesoporous silica surface after submonolayer deposition of aminopropylsilanes from an anhydrous liquid phase. J Phys Chem C 111:923-929

30. Lim MH, Stein A (1999) Comparative studies of grafting and direct syntheses of inorganic-organic hybrid mesoporous materials. Chem Mater 11:3285-3295

31. Acosta EJ, Carr CS, Simanek EE, Shantz DF (2004) Engineering nanospaces: iterative synthesis of melamine-based dendrimers on amine-functionalized SBA-15 leading to complex hybrids with controllable chemistry and porosity. Adv Mater 16:985-989

32. Fukuoka A, Fujishima K, Chiba M, Yamagishi A, Inagaki S, Fukushima Y, Ichikawa M (2000) Photooxidation of cyclohexene and benzene with oxygen by fullerenes grafted on mesoporous FSM-16. Catal Lett 68:241-244

33. Burkett SL, Sims SD, Mann S (1996) Synthesis of hybrid inorganic-organic mesoporous silica by co-condensation of siloxane and organosiloxane precursors. Chem Commun 11:1367-1368

34. Macquarrie DJ (1996) Direct preparation of organically modified MCM-type materials. Preparation and characterisation of aminopropyl-MCM and 2-cyanoethyl-MCM. Chem Commun 16:1961-1962

35. Mercier L, Pinnavaia TJ (2000) Direct synthesis of hybrid organic-inorganic nanoporous silica by a neutral amine assembly route: structure-function control by stoichiometric incorporation of organosiloxane molecules. Chem Mater 12:188-196

36. Huh S, Wiench JW, Yoo J-C, Pruski M, Lin VS-Y (2003) Organic functionalization and morphology control of mesoporous silicas via a co-condensation synthesis method. Chem Mater 15:4247-4256

37. Fowler CE, Burkett SL, Mann S (1997) Synthesis and characterization of ordered organo-silica-surfactant mesophases with functionalized MCM-41-type architecture. Chem Mater 18:1769-1770

38. Cagnol F, Grosso D, Sanchez C (2004) A general one-pot process leading to highly functionalised ordered mesoporous silica films. Chem Commun 10:1742-1743
39. Inagaki S, Guan S, Fukushima Y, Ohsuna T, Terasaki O (1999) Novel mesoporous materials with a uniform distribution of organic groups and inorganic oxide in their frameworks. J Am Chem Soc 121:9611-9614

40. Melde BJ, Holland BT, Blanford CF, Stein A (1999) Mesoporous sieves with unified hybrid inorganic/organic frameworks. Chem Mater 11:3302-3308

41. Asefa T, MacLachlan MJ, Coombs N, Ozin GA (1999) Periodic mesoporous organosilicas with organic groups inside the channel walls. Nature 402:867-871

42. Yoshina-Ishii C, Asefa T, Coombs N, MacLachlan MJ, Ozin GA (1999) Periodic mesoporous organosilicas, PMOs: fusion of organic and inorganic chemistry 'inside' the channel walls of hexagonal mesoporous silica. Chem Commun 24:2539-2540

43. Kapoor MP, Yang Q, Inagaki S (2002) Self-assembly of biphenylene-bridged hybrid mesoporous solid with molecularscale periodicity in the pore walls. J Am Chem Soc 124:15176-15177

44. Kim D-J, Chung J-S, Ahn W-S, Kang G-W, Cheong W-J (2004) Morphology control of organic-inorganic hybrid mesoporous silica by microwave heating. Chem Lett 33:422-423

45. Fukuoka A, Sakamoto Y, Guan S, Inagaki S, Sugimoto N, Fukushima Y, Hirahara K, Iijima S, Ichikawa M (2001) Novel templating synthesis of necklace-shaped mono- and bimetallic nanowires in hybrid organic-inorganic mesoporous material. J Am Chem Soc 123:3373-3374

46. Boltz DF, Howell JA (1978) Colorimetric determination of nonmetals, vol 8, 2nd edn. Wiley, New York

47. Chang F-Y, Chao K-J, Cheng H-H, Tan C-S (2009) Adsorption of $\mathrm{CO}_{2}$ onto amine-grafted mesoporous silicas. Sep Purif Technol 70:87-95

48. Seçkin T, Gültek A, Kartaca S (2003) The grafting of Rhodamine B onto sol-gel derived mesoporous silicas. Dyes Pigments 56:51-57

49. Rosenholm JM, Czuryszkiewicz T, Kleitz F, Rosenholm JB, Lindén M (2007) On the nature of the Brønsted acidic groups on native and functionalized mesoporous siliceous SBA-15 as studied by benzylamine adsorption from solution. Langmuir 23:4315-4323 